\title{
A COMMON FIXED POINT THEOREM FOR A SEQUENCE OF FUZZY MAPPINGS
}

\author{
B. E. RHOADES \\ Department of Mathematics \\ Indiana University \\ Bloomington, Indiana 47405, U.S.A. \\ (Received September 29, 1994)
}

ABSTRACT. We obtain a common fixcd point theorem for a sequence of fuzzy mappings, satisfying a contractive definition more general than that of Lee, Lee, Cho and Kim [2].

Let $(X, d)$ be a completc linear metric space. A fuzzy set $A$ in $X$ is a function from $X$ into $[0,1]$. If $x \in X$, the function value $A(x)$ is called the grade of membership of $X$ in $A$. The $\alpha$-level set of $A, A_{\alpha}:=\{x: A(x) \geq \alpha$, if $\alpha \in(0,1]\}$, and $A_{0}:=\overline{\{x: A(x)>0\}} . W(X)$ denotes the collection of all the fuzzy sets $A$ in $X$ such that $A_{\alpha}$ is compact and convex for each $\alpha \in[0,1]$ and $\sup _{x \in X} A(x)=1$. For $A, B \in W(X), A \subset B$ means $A(x) \leq B(x)$ for each $x \in X$. For $A, B \in W(X), \alpha \in[0,1]$, define

$$
P_{\alpha}(A, B)=\inf _{x \in A_{\alpha}, y \in B_{\alpha}} d(x, y), P(A, B)=\sup _{\alpha} P_{\alpha}(A, B), D(A, B)=\sup _{\alpha} d_{H}\left(A_{\alpha}, B_{\alpha}\right),
$$

where $d_{H}$ is the Hausdorff metric induced by the metric $d$. We note that $P_{\alpha}$ is a nondecreasing function of $\alpha$ and $D$ is a metric on $W(X)$.

Let $X$ be an arbitrary set, $Y$ any linear metric space. $F$ is called a fuzzy mapping if $F$ is a mapping from the set $X$ into $W(Y)$.

In earlier papers the author and Bruce Watson, [3] and [4], proved some fixed point theorems for some mappings satisfying a very general contractive condition. In this paper we prove a fixed point theorem for a sequence of fuzzy mappings satisfying a special case of this general contractive condition. We shall first prove the theorem, and then demonstrate that our definition is more general than that appearing in [2].

Let $D$ denote the closure of the range of $d$. We shall be concerned with a function $Q$, defined on $d$ and satisfying the following conditions:

(a) $0<Q(s)<s$ for each $s \in D \backslash\{0\}$ and $Q(0)=0$,

(b) $Q$ is nondecreasing on $D$, and

(c) $g(s):=s /(s-Q(s))$ is nonincreasing on $D \backslash\{0\}$.

LEMMA 1. [1] Let $(X, d)$ be a complete linear metric space, $F$ a fuzzy mapping from $X$ into $W(X)$ and $x_{0} \in X$. Then there exists an $x_{1} \in X$ such that $\left\{x_{1}\right\} \subset F\left(x_{0}\right)$. 
THEOREM 1. Let $g$ be a nonexpansive selfmap of $X,(X, d)$ a complete linear metric space. Let $\left\{F_{\imath}\right\}$ be a sequence of fuzzy mappings from $X$ into $W(X)$ satisfying: For cach pair of fuzzy mappings $F_{2}, F_{3}$ and for any $x \in X,\left\{u_{x}\right\} \subset F_{2}(x)$, there exists a $\left\{v_{y}\right\} \subset F_{j}(y)$ for all $y \in X$ such that

$$
D\left(\left\{u_{x}, v_{y}\right\}\right) \leq Q(m(x, y))
$$

where

$$
\begin{aligned}
m(x, y):=\max \{ & d\left(g(x), g\left(u_{x}\right)\right), d\left(g(y), g\left(v_{y}\right)\right),\left[d\left(g(y), g\left(u_{x}\right)\right)\right. \\
& \left.\left.+d\left(g(x), g\left(v_{y}\right)\right)\right] / 2, d(g(x), g(y))\right\}
\end{aligned}
$$

and $Q$ satisfies (a) - (c). Then there exists a $p \in \cap_{\imath=1}^{\infty} F_{\imath}(p)$.

PROOF. Let $x_{0} \in X$. Then we can choose $x_{1} \in X$ such that $\left\{x_{1}\right\} \subset F_{1}\left(x_{0}\right)$ by Lemma 1 . From the hypothesis, there exists an $x_{2} \in X$ such that $\left\{x_{2}\right\} \subset F_{2}\left(x_{1}\right)$ and, from (1),

$$
\begin{aligned}
D\left(\left\{x_{1}\right\},\left\{x_{2}\right\}\right) \leq & Q\left(m\left(x_{1}, x_{2}\right)\right) \\
< & \max \left\{d\left(g\left(x_{0}\right), g\left(x_{1}\right)\right), d\left(g\left(x_{1}, g\left(x_{2}\right)\right),\right.\right. \\
& \left.\quad\left[d\left(g\left(x_{1}\right), g\left(x_{1}\right)\right)+d\left(g\left(x_{0}\right), g\left(x_{2}\right)\right)\right] / 2, d\left(g\left(x_{0}\right), g\left(x_{1}\right)\right)\right\} \\
\leq & \max \left\{d\left(x_{0}, x_{1}\right), d\left(x_{1}, x_{2}\right),\left[d\left(x_{1}, x_{1}\right)+d\left(x_{0}, x_{2}\right)\right] / 2, d\left(x_{0}, x_{1}\right)\right\},
\end{aligned}
$$

since $g$ is nonexpansive.

Inductively, we obtain a sequence $\left\{x_{n}\right\}$ such that $x_{n+1} \subset F_{n+1}\left(x_{n}\right)$ and

$$
\begin{aligned}
D\left(\left\{x_{n}\right\},\left\{x_{n+1}\right\}\right) \leq & Q\left(m\left(x_{n}, x_{n+1}\right)\right) \\
<\max \{ & \left.d\left(x_{n-1}, x_{n}\right), d\left(x_{n}, x_{n+1}\right),\left[d\left(x_{n} x_{n}\right)+d x_{n-1}, x_{n+1}\right)\right] / 2, \\
& \left.d\left(x_{n-1}, x_{n}\right)\right\} .
\end{aligned}
$$

Since $D\left(\left\{x_{n}\right\},\left\{x_{n+1}\right\}\right)=d\left(x_{n}, x_{n+1}\right)$, it follows from (3) that $d\left(x_{n}, x_{n+1}\right)<d\left(x_{n-1}, x_{n}\right)$. Using this fact back in (2), we obtain that $d\left(x_{n}, x_{n+1}\right) \leq d\left(x_{n-1}, x_{n}\right)$. Substituting into (3) we obtain

$$
d\left(x_{n}, x_{n+1}\right)<Q\left(d\left(x_{n-1}, x_{n}\right)\right)<Q^{2}\left(d\left(x_{n-2}, x_{n-1}\right)<\cdots<Q^{n}\left(d\left(x_{0}, x_{1}\right)\right) .\right.
$$

From Lemma 2 of [3], $\lim Q^{n}\left(d\left(x_{0}, x_{1}\right)\right)=0$. To show that $\left\{x_{n}\right\}$ is Cauchy, choose $N$ so large that $Q^{n}\left(d\left(x_{0}, x_{1}\right)\right) \leq 1 / 2$ for all $n>N$. Then, for $m>n>N$,

$$
d\left(x_{n}, x_{m}\right) \leq \sum_{i=n}^{m-1} d\left(x_{\imath}, x_{\imath+1}\right) \leq \sum_{i=n}^{m-1} Q^{2}\left(d\left(x_{0}, x_{1}\right)\right) \leq \sum_{i=n}^{m-1}\left(\frac{1}{2}\right)^{2}<\frac{1}{2^{n}}\left(1-\frac{1}{2}\right),
$$

and $\left\{x_{n}\right\}$ is Cauchy, hence convergent. Call the limit $p$.

Let $F_{m}$ be an arbitrary member of the sequence $\left\{F_{2}\right\}$. Since $\left\{x_{n}\right\} \subset F_{n}\left(x_{n-1}\right)$, there exists a $v_{n} \in X$ such that $\left\{v_{n}\right\} \subset F_{m}(p)$ for all $n$ and

$$
\begin{aligned}
D\left(\left\{x_{n}\right\},\left\{v_{n}\right\}\right) \leq & Q\left(m\left(x_{n}, v_{n}\right)\right) \\
& =Q\left(\operatorname { m a x } \left\{d\left(g\left(x_{n-1}\right), g\left(x_{n}\right)\right), d\left(g(p), g\left(v_{n}\right)\right),\right.\right. \\
& \left.\left.\quad\left[d\left(g\left(x_{n-1}\right), g\left(v_{n}\right)\right)+d\left(g(p), g\left(x_{n}\right)\right)\right] / 2, d\left(g\left(x_{n-1}\right), g(p)\right)\right\}\right) \\
\leq & Q\left(\max \left\{d\left(x_{n-1}, x_{n}\right), d\left(p, v_{n}\right),\left[d\left(x_{n-1}, v_{n}\right)+d\left(p, x_{n}\right)\right] / 2, d\left(x_{n-1}, p\right)\right\}\right) .
\end{aligned}
$$

Suppose that $\lim v_{n} \neq p$. Taking the limit as $n \rightarrow \infty$ yields, since $Q$ is continuous (Lemma 1 of $[3])$,

$$
\limsup d\left(p, v_{n}\right) \leq Q\left(\limsup d\left(p, v_{n}\right) \dot{<} \lim \sup d\left(p, v_{n}\right),\right.
$$


a contradiction. Therefore $\lim v_{n}=p$. Since $F_{m}(p) \in W(X), F_{m}(p)$ is upper semicontinuous and therefore $\lim \sup \left[F_{m}(p)\right]\left(v_{n}\right) \leq\left[F_{m}(p)\right](p)$. Since $\left\{v_{n}\right\} \subset F_{m}(p)$ for all $n,\left[F_{m}(p)\right](p)=1$. Hence $\{p\} \subset F_{m}(p)$. Since $F_{m}$ is arbitrary, $\{p\} \subset \cap_{\imath=1}^{\infty} F_{\imath}(p)$.

The contractive definition of $[2]$ is the following :

$$
\begin{gathered}
D\left(\left\{u_{x}\right\},\left\{v_{y}\right\}\right) \leq a_{1} d\left(g(x), g\left(u_{x}\right)\right)+a_{2} d\left(g(y), g\left(v_{y}\right)\right)+a_{3} d\left(g(y), g\left(u_{x}\right)\right)+ \\
a_{4} d\left(g(x), g\left(v_{y}\right)\right)+d(g(x), g(y))
\end{gathered}
$$

where each $a_{2}$ is nonnegative, $\sum_{2=1}^{5} a_{2}<1$, and $a_{3} \geq a_{4}$.

In (4), if one interchanges the roles of $x$ and $y$ one obtains

$$
\begin{gathered}
\left.D\left(\left\{v_{y}\right\},\left\{u_{x}\right\}\right) \leq a_{1} d\left(g(y), v_{y}\right)\right)+a_{2} d\left(g(x), g\left(u_{x}\right)\right)+a_{3} d\left(g(x), g\left(v_{y}\right)\right)+ \\
a_{4} d\left(g(y), g\left(u_{x}\right)\right)+a_{5} d(g(y), g(x)) .
\end{gathered}
$$

Adding (4) and (5) yiclds

$$
\begin{gathered}
D\left(\left\{u_{x}\right\},\left\{v_{y}\right\}\right) \leq \alpha_{1} d\left(g(x), g\left(u_{x}\right)\right)+\alpha_{2} d\left(g(y), g\left(v_{y}\right)\right)+\alpha_{3} d\left(g(y), g\left(u_{x}\right)\right)+ \\
\alpha_{1} d\left(g(x), g\left(v_{y}\right)\right)+\alpha_{5} d(g(x), g(y))
\end{gathered}
$$

where $\alpha_{1}=\alpha_{2}=\left(a_{1}+a_{2}\right) / 2, \alpha_{3}=\alpha_{4}=\left(a_{3}+a_{4}\right) / 2$, and $\alpha_{5}=a_{5}$. In turn, (6) implies that

$$
\begin{aligned}
D\left(\left\{u_{x}\right\},\left\{v_{y}\right\}\right) \leq & \left(\alpha_{1}+\alpha_{2}\right) \max \left\{d\left(g(x), g\left(u_{x}\right)\right), d\left(g(y), g\left(v_{y}\right)\right)\right\}+ \\
& \alpha_{3}\left[d\left(g(y), g\left(u_{x}\right)\right)+d\left(g(x), g\left(v_{y}\right)\right)\right] / 2+\alpha_{5} d(g(x), g(y)) \\
\leq & \left(\alpha_{1}+\alpha_{2}+\alpha_{3}+\alpha_{5}\right) m(x, y)=\left(\sum_{z=1}^{5} a_{\imath}\right) m(x, y)=h m(x, y)
\end{aligned}
$$

say.

(7) is the special case of (1) with $Q(s)=h s$. Consequently Theorem 3.1 of [2], as well as the corollaries, are special cases of the theorem of this paper.

\section{REFERENCES}

1. LEE, B. S. and CHO, S. J., A fixed point theorem for contractive-type fuzzy mappings, Fuzzy Sets and Systems (submitted).

2. LEE, B. S., LEE, G. M., CHO, S. J. and KIM, D. S., Generalized common fixed point theorems for a sequence of fuzzy mappings, Internat. J. Math. \& Math. Sci. 17(1994), 437-440.

3. RHOADES, B. E. and WATSON, B., Generalized contractions and fixed points in metric spaces, Math. Japonica 34(1989), 975-982.

4. RHOADES, B. E. and WATSON, B., Fixed points for set-valued mappings in metric spaces, Math. Japonica 35(1990), 735-743. 


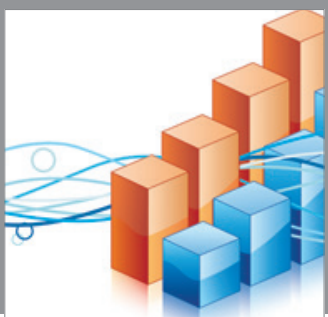

Advances in

Operations Research

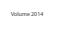

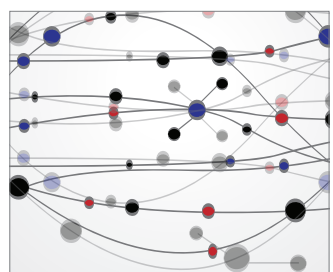

\section{The Scientific} World Journal
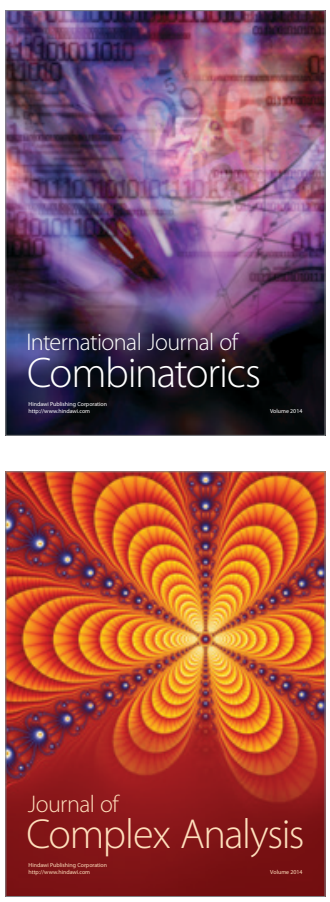

International Journal of

Mathematics and

Mathematical

Sciences
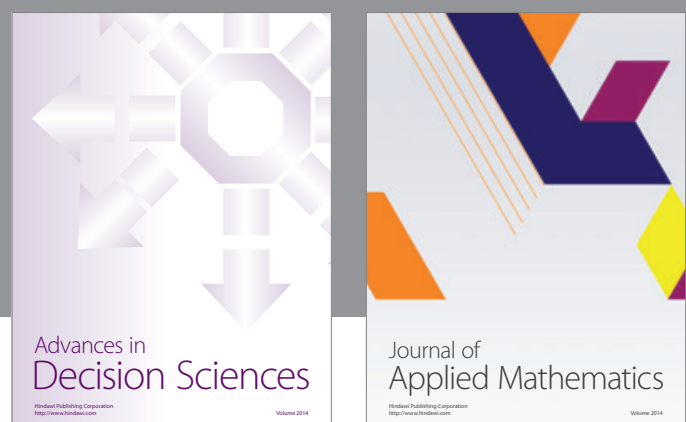

Journal of

Applied Mathematics
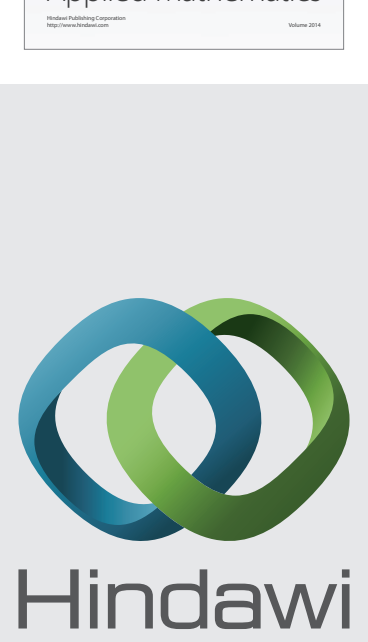

Submit your manuscripts at http://www.hindawi.com
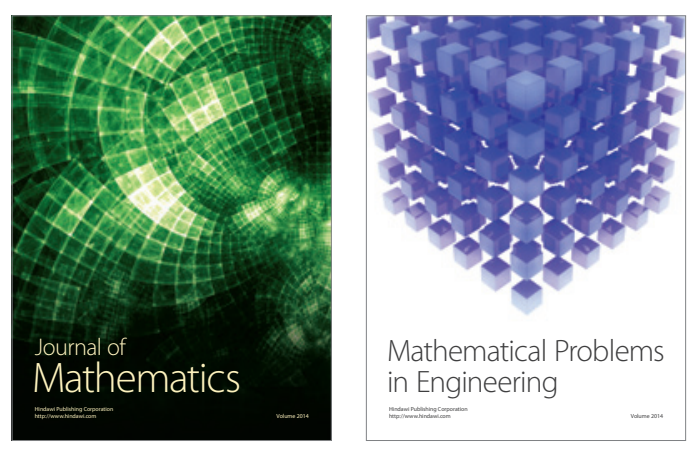

Mathematical Problems in Engineering
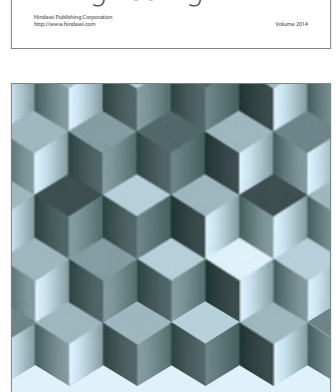

Journal of

Function Spaces
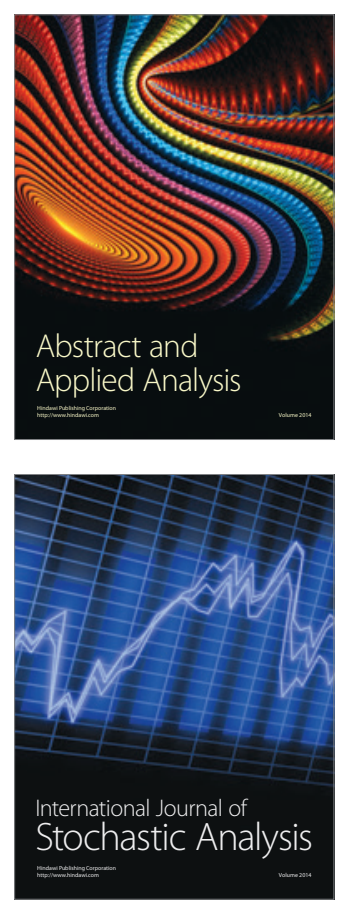

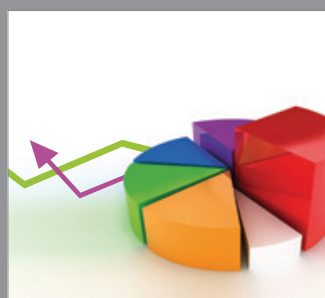

ournal of

Probability and Statistics

Promensencen
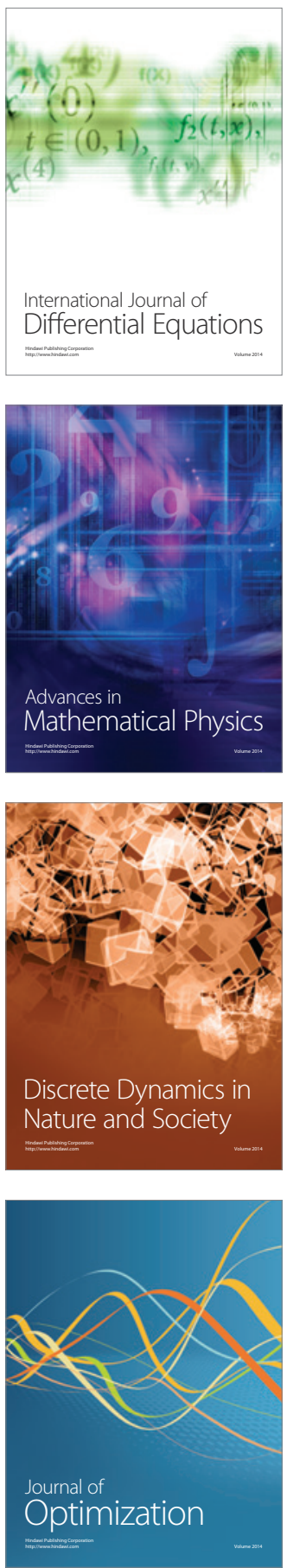